Revue internationale d'éthique sociétale et gouvernementale

vol. 18, n 2 | 2016

Éthique et scandales publics

\title{
Des cris au silence médiatique : les limites de la scandalisation
}

Pierre Lascoumes

revues.org

Édition électronique

URL : http://

ethiquepublique.revues.org/2799

DOI : $10.4000 /$ ethiquepublique.2799

ISSN : 1929-7017

\section{Éditeur \\ Éditions Nota bene}

Édition imprimée

Date de publication : 1 septembre 2016

ISSN : 1488-0946

Ce document vous est offert par Sciences

Po.

\section{SciencesPo.}

Référence électronique

Pierre Lascoumes, «Des cris au silence médiatique : les limites de la scandalisation », Éthique publique [En ligne], vol. 18, n² 2 | 2016, mis en ligne le 23 janvier 2017, consulté le 03 février 2017. URL http://ethiquepublique.revues.org/2799; DOI : 10.4000/ethiquepublique.2799

Ce document a été généré automatiquement le 3 février 2017

Tous droits réservés 


\title{
Des cris au silence médiatique : les limites de la scandalisation
}

\author{
Pierre Lascoumes
}

1 La rhétorique de la dénonciation des « scandales » est aussi banale que puissante. Mais les effets de la mise en accusation publique restent aléatoires ${ }^{1}$. Certes, les médias de divertissement ont de longue date mis en scène les comportements transgressifs des élites dans leur milieu professionnel. Des films ont obtenu de grands succès : du scandale du Watergate (All the presidents'men, 1976), à la débâcle financière de 2008 (Too big to fail, 2011), en passant par la dénonciation de la corruption (All the King's men, 1949; The best man, 1964 ; City Hall, 1996; Lord of War, 2005). Des séries télévisées font de ces sujets la matière récurrente d'excellents scénarios : Boss, Boardwalk Empire, The Wire. Mais le plaisir de l'indignation morale suscité par des fictions est très éloigné des vicissitudes des réformes sociales et politiques.

2 À la suite de différentes enquêtes sur la réaction sociale aux déviances et délinquances des élites - matière favorite de la scandalisation -, j'ai montré le contraste massif qui existe dans la durée entre la virulence initiale des campagnes médiatiques de dénonciation et la faiblesse des réactions sociales finales. Chaque fois qu'est révélé un évènement montrant un problème de transgression majeure des normes sociales impliquant un dirigeant (public ou privé), la situation est présentée comme intolérable. Le terme "scandale " est très facilement utilisé. Nombre de journalistes et d'experts appellent à des sanctions exemplaires et à des mesures de prévention exigeantes. Mais après des mois, régulièrement après des années de silence, le "scandale " n'est plus qu'une « affaire» et se trouve réduit finalement à un «problème technique » dépourvu d'enjeu majeur. Souvent, aucune poursuite n'a lieu, l'affaire est classée faute de preuve ou de gravité démontrée. Au bout du compte, il y a peu ou pas de sanction et peu ou pas de grande réforme. Ainsi, longue est la liste des «scandales " à court terme dont l'issue judiciaire et politique a été purement symbolique, ou bien elle s'est effilochée dans les méandres des expertises et de la procédure. Les procès du Crédit lyonnais (de Blic, 2005) 
ou de l'ex-président Jacques Chirac (Lascoumes, 2013) en fournissent des exemples significatifs.

\section{Derrière le « scandale », des catégorisations plurielles}

3 La notion de scandale demeure une catégorie floue et toujours objet de controverse (de Blic et Lemieux, 2005). Dans une étude pionnière, Éric de Dampierre écrit : « le scandale est $1^{\circ}$ une occasion de chute, de péché, et $2^{\circ}$ due à l'action ou au discours de quelqu'un. Deux personnages sont donc en scène, le scandalisé et le scandalisant» (1954: 329). Depuis, tous les travaux sur le sujet s'accordent pour reconnaître qu'une accusation publique ne devient un scandale que si deux conditions sont réunies: d'un côté, une transgression significative de normes et de valeurs reconnues ayant une fonction symbolique dans la conception dominante d'un ordre social, local ou global; d'un autre côté, une publicisation qui est à la fois un processus de qualification particulier de l'acte et une divulgation suivie de mobilisation d'une audience. Cette deuxième dimension du scandale est considérée aujourd'hui comme déterminante. Dans sa théorie générale du scandale, John. B. Thompson accorde aux différents degrés de publicisation une place déterminante $(2000)^{2}$. Ces approches reprennent une perspective interactionniste tempérée en considérant qu'au-delà d'une action initiale d'atteinte à une norme, l'essentiel s'accomplit par des dynamiques de réaction sociale. Elles attribuent alors à la mobilisation et à l'interprétation de l'évènement et de ses conséquences un rôle plus décisif qu'aux caractéristiques de l'acte transgressif initial (l'auteur, le contexte, l'ampleur). La mise en accusation publique qu'est le scandale résulte donc d'une série d'activités sociales qu'il s'agit de décrypter. Ces auteurs se sont attachés à dénaturaliser la notion de scandale, en montrant les stratégies d'acteurs dans lesquelles il intervient, et en particulier les liens qui existent entre compétition politique et accusation publique ${ }^{3} . \mathrm{H}$. Tumber et S. R. Waisborg (2004) dans leur travail de comparaison internationale font de la médiatisation un trait distinctif de la production des scandales politiques dans les sociétés démocratiques. Certains auteurs tels de Blic estiment cependant qu'une importance trop grande donnée aux activités de construction sociale de la réalité peut devenir réductrice et qu'il est possible d'établir « une proportionnalité entre la gravité de la transgression et l'importance du scandale » (de Blic, 2005).

4 Malgré ces différences d'approche plus ou moins marquées, ces travaux se rejoignent cependant dans ce que j'appelle un «globalisme », c'est-à-dire dans une faible attention portée aux différences d'interprétation données à un même évènement selon les locuteurs et selon les séquences de déroulement temporel. Dans ces raisonnements, tout semble en effet se passer comme si la transgression qui sert de base à la mobilisation accusatrice pouvait être lue de façon homogène par tous les acteurs sociaux quels qu'ils soient. Chaque situation parait porteuse d'une signification sinon unique, du moins largement dominante et qui s'imposerait de facto. Cela conduit à parler hâtivement au singulier de "l'affaire $\mathrm{X}$ » et du "scandale $\mathrm{Z}$ » indépendamment des appartenances sociales et des attitudes politiques des énonciateurs. Quelques travaux ont au contraire posé l'hypothèse d'une pluralité des constructions effectuées à partir d'un même évènement.

5 Une recherche faite sur les qualifications données à une fraude sur les appellations des vins de Bordeaux réalisée par des négociants a montré comment une dénonciation initiale véhémente a été progressivement fragmentée, certains intervenants reprenant de plus en 
plus une argumentation technique sur les ambiguïtés des «appellations viticoles contrôlées ». À l'occasion du procès, les divergences ont été accentuées, certains allant jusqu'à retourner l'accusation contre les excès de zèle d'une " administration tatillonne " qui nuirait à l'image du vignoble bordelais et porterait atteinte à « l'équilibre économique de la région » (Armand et Lascoumes, 1977). Cette hétérogénéité dans les perceptions est liée à la diversité des groupes sociaux et des représentations du monde dont ils sont porteurs. Il importe de différencier les formes d'accusation publique, de souligner ce qui les distingue et de montrer quelles dimensions elles privilégient dans leur lecture des évènements sociaux. Chaque acte transgressif est un enjeu de qualification, d'où l'importance du repérage des structures discursives concurrentes qui coexistent. Parler d'accusation, de dénonciation publique ou de scandalisation c'est selon l'expression de Damien de Blic (2003) s'intéresser à une "mise en forme de l'évènement public ». Comment alors distinguer ces formes et comprendre leur variété ? Dans ses travaux sur la dénonciation publique, Luc Boltanski (1990: 255-356) fait de la notion "d'affaire » la forme de base de la défense des causes. Il la définit comme un « processus d'enrôlement autour d'un cas problématique et litigieux dont la détermination et la décision sont liées aux manœuvres argumentatives et probatoires, et aux efforts de mobilisation déployés dans chaque camp » (1990: 256). Il distingue deux modalités antagonistes, l'une visant à "grandir ", "grossir ", (faire) "sortir » l'affaire; l'autre en sens inverse visant à la « ramener à de justes proportions", la « dégonfler », l'« étouffer dans l'œuf ». Selon lui ces opérations «sont ainsi toujours associées à des déplacements, entre le "cas particulier " et l'"intérêt général", le "singulier et le collectif" " $(1990: 256)$. Cette proposition a été mise à l'épreuve sur des matériaux très différents afin d'analyser comment s'édifient des causes et se construit leur carrière ${ }^{4}$. Il est cependant possible d'aller plus avant dans cette différenciation, en particulier pour l'analyse des représentations produites par les médias. Pour préciser les dynamiques relevées par Boltanski d'amplification versus de banalisation applicables à une situation problématique, nous proposons trois formes de qualification publique. Le tableau ci-dessous en synthétise les principales dimensions qui organisent la structure argumentative des formes " problème », « affaire » et « scandale » (Tableau 1).

6 C'est dans un travail antérieur sur le dossier Elf (Lascoumes, 1997) que nous avions dégagé ces trois répertoires de qualification d'un évènement. L'accusation publique se différencie selon quatre grandes composantes. 1 - Le rapport aux normes : existe-t-il des normes cadrant, et jusqu'où, la situation? Dans quelle mesure estime-t-on que des règles ont été ou non transgressées? La violation est-elle accidentelle ou délibérée ? 2- Les bénéfices : des profits illicites ont-ils été réalisés? Si oui quel est leur montant? Y a-t-il des victimes et des dommages? Sont-ils généraux ou bien spécifiés? Quelle évaluation peut-on en faire ? 3 - Les responsabilités : sont-elles établies ? Peut-on attribuer la prise de décision ayant généré l'évènement mis en cause à un ou des acteurs précis ? Ou bien at-on affaire à un réseau plus ou moins structuré ? Ou encore à une série de micro-choix dont l'enchaînement était imprévisible? Au bout du compte peut-on parler ou non d'intention coupable? 4 - La réaction sociale attendue : quelles conséquences devraient avoir la situation révélée ? Un effet d'apprentissage, l'adoption de mesures de réparation ou le prononcé de sanction? On peut considérer que ces trois modèles sont des idéauxtypes au sens wébérien qu'il ne s'agit pas de chercher à valider à la lettre par des données empiriques. Mais ils permettent de définir une grille analytique de base et de poser des hypothèses sur le type de mise en accusation que l'on cherche à comprendre. 
7 Le Tableau 1 présente les trois répertoires selon un continuum où la gravité et potentiellement la réprobation vont du plus faible au plus fort. Mais ce n'est qu'une présentation à vertu pédagogique. Nous voulons insister au contraire sur le fait qu'un évènement ne relève pas par nature ou constitutivement de telle ou telle catégorie. Au contraire, nous traitons ici de dynamiques de qualification. Selon les périodes, les locuteurs peuvent s'appuyer sur tel ou tel registre sans respecter un ordre croissant ou décroissant. Ce qui n'est qu'un " problème » dans un temps 1 (le flou dans une procédure d'attribution de marché public), peut soudainement devenir un "scandale » (un parti politique a tiré avantage de l'incertitude pour favoriser des soutiens), et finalement après enquête n'être qu'une "affaire " (seul un petit groupe de dirigeants ont pendant une période limitée tiré avantage des lacunes juridiques. Depuis, les règles ont été clarifiées).

Tableau $1:$ Trois formes de mise en accusation publique et leurs composantes

\begin{tabular}{|c|c|c|c|}
\hline $\begin{array}{l}\text { Qualification de } \\
\text { l'accusation publique } \\
\text { et ses composantes }\end{array}$ & Problème & Affaire & Scandale \\
\hline $\begin{array}{l}\text { Violation de normes } \\
\text { sociales }\end{array}$ & Très incertaine & Conflits normatifs & Caractérisée \\
\hline $\begin{array}{l}\text { Obtention de bénéfices } \\
\text { illicites } \\
\text { Importance } \\
\text { victimes et des } \\
\text { dommages }\end{array}$ & $\begin{array}{l}\text { Pas de bénéfices } \\
\text { illicites } \\
\text { Dommages } \\
\text { inexistants ou } \\
\text { inévaluables }\end{array}$ & $\begin{array}{l}\text { Des bénéfices ont été } \\
\text { réalisés. Leur licéité est } \\
\text { controversée } \\
\text { Victimes identifiées et/ } \\
\text { ou dommages } \\
\text { évaluables }\end{array}$ & $\begin{array}{l}\text { Obtention de bénéfices } \\
\text { illicites } \\
\text { Victimes très visibles } \\
\text { et/ou dommages très } \\
\text { importants }\end{array}$ \\
\hline $\begin{array}{l}\text { Degré } \quad \text { de } \\
\text { responsabilité }\end{array}$ & $\begin{array}{l}\text { Responsabilités non } \\
\text { établies }\end{array}$ & $\begin{array}{l}\text { Responsabilités } \\
\text { incertaines, } \\
\text { entremêlées }\end{array}$ & $\begin{array}{l}\text { Responsabilités } \\
\text { attribuées }\end{array}$ \\
\hline $\begin{array}{l}\text { Réaction } \quad \text { sociale } \\
\text { attendue }\end{array}$ & Absente & Faible & Forte \\
\hline
\end{tabular}

\section{«Pechiney-Triangle » : scandale, affaire ou problème?}

8 Nous allons prendre maintenant l'exemple d'un dossier à caractère politico-financier pour montrer les fluctuations dans les appréciations portées par les médias. Si ce dossier a pu faire «scandale", encore faut-il préciser lequel et pour qui ? Pour plusieurs locuteurs, il n'a jamais été qu'un " problème » de régulation boursière. Pour d'autres, les avantages obtenus par des acteurs politiques très proches des acteurs décisionnaires donnent de bout en bout à ce dossier le caractère d'une « affaire ».

9 À l'occasion de l'achat par l'entreprise métallurgique publique Pechiney de la société étasunienne Triangle une importante spéculation boursière illicite (délit d'initié) a eu lieu entre août et novembre 1988. Elle a suscité une des plus importantes mises en accusation publiques de l'élite dirigeante des années 1990. Les négociations entre les deux 
entreprises débutent en juin 1988 et s'achèvent le 21 novembre. Des mouvements d'actions a-typiques accompagnent les fluctuations des échanges. Surtout, des achats importants sont opérés durant la période de négociation finale, 330000 titres sont ainsi échangés entre les 14 et 18 novembre. Des reventes massives suivent immédiatement la montée du cours en novembre et décembre 1988. Les opérateurs qui agissent ainsi triplent ou quadruplent leur mise (en millions de francs). Le scandale éclate en janvier 1989 avec la révélation par le journal Le Monde des opérations et gains dégagés par Patrice Pelat, un proche du président de la République François Mitterrand. D'autres bénéficiaires en proximité avec le ministère des Finances où se négocie le dossier sont mis en cause. Tous les éléments se trouvent ici réunis pour déclencher une mobilisation sans précédent: une transgression avérée, des profits illicites massifs, des acteurs économiques et politiques de premier plan, une révélation par la presse, enfin, une réaction ferme des autorités de contrôle et de sanction. Pour le gouvernement socialiste de l'époque, le dossier "Pechiney-Triangle» fut une situation délicate à gérer dans la mesure où elle s'ajoutait aux « affaires » de financement politique déjà en cours. Il a pris à plusieurs reprises et pour certains acteurs l'ampleur d'un «scandale » déstabilisant les institutions et menaçant de susciter une « crise » politique.

Nous avons travaillé à partir d'un corpus de 302 articles de presse concernant les six principaux quotidiens de la presse nationale française ${ }^{6}$. Une analyse effectuée avec le logiciel d'analyse de texte Alceste ${ }^{7}$ a permis de dégager les registres discursifs des discours de presse et leur évolution (Hamidi, 2009). Huit classes d'arguments ont été regroupées en trois grandes catégories: une dimension économique (35\%) (transaction industrielle, opération boursière, circuits offshore, traders) ; une dimension politique (33\%) (acteurs politiques, réseaux politico-financiers); et une dimension de réaction sociale (32\%) (enquête de la Commission des opérations de bourse, poursuites judiciaires). Deux résultats principaux ont été dégagés.

11 En observant sur six ans l'évolution du nombre d'articles publiés, nous avons distingué trois périodes. Ils correspondant aux temps forts de l'affaire : 1988-1989, la révélation et la découverte progressive des implications ; 1990-1992, l'instruction ; 1993, le procès. L'analyse séquentielle montre clairement l'effritement du traitement politique et d'une dénonciation en termes de scandale au profit des dimensions techniques économiques et judiciaires.

- Durant la période initiale de révélation des faits (1988-1989), les dimensions politiques et économiques se partagent l'essentiel de la couverture par la presse (chacune $38 \% \mathrm{du}$ corpus), tandis que la dimension d'enquête compte pour une part plus faible, avec $24 \% \mathrm{du}$ corpus. Si pour certains médias il s'agit d'un «scandale d'État» impliquant les plus hauts responsables, pour tous les autres (y compris la presse économique) il y a au moins une « affaire » car des bénéfices suspects ont été réalisés. Leur licéité est controversée, s'agit-il ou non d'un usage abusif d'informations privilégiées ou bien d'une pratique boursière ordinaire basée sur des paris qui ont réussi?

- Durant la deuxième période (1990-1992), la dimension politique disparaît presque complètement, la reconstitution des faits par les enquêtes administratives et judiciaires (42\%) et surtout les interprétations économiques de l'opération (58 \%) prévalent. Même les journaux qui utilisaient le plus le répertoire de dénonciation du scandale modèrent leurs arguments et commencent à relativiser la gravité des faits. L'élargissement des informations que permet l'avancée des enquêtes, ainsi que la mise évidence d'incertitudes dans les 
processus de décision et leurs méandres, conduisent à utiliser de plus en plus le registre de type « Affaire».

- Enfin, durant la troisième période (1993), celle du procès, la dimension politique fait un retour, mais elle reste limitée (17\%). Aucun acteur politique n'étant renvoyé devant les juges, le registre "scandale» est peu repris. Ce sont les circuits de circulation des informations économiques et boursières sur l'opération qui sont débattus. Ils s'avèrent touffus en raison de l'ampleur financière de l'opération et de sa réalisation sur deux continents. Des acteurs industriels et bancaires y côtoient les conseillers économiques et diplomatiques des ministres et du président. En revanche, les deux autres dimensions (Économie et Enquête) décroissent un peu (respectivement, 30 et $52 \%$ ) mais continuent à fournir l'essentiel des arguments.

12 Un deuxième résultat montre la diversité des traitements journalistiques. Le Monde est le seul à avoir eu un traitement presque continu en termes de "scandale ". C'est lui qui révèle la spéculation boursière et il met en cause d'entrée les responsables politiques (proches du président de la République et cabinet du ministère des Finances). Durant la deuxième période, la " titraille » de ce journal ${ }^{8}$ reste dans ce registre, mais le contenu des articles fait place aux incertitudes sur les circuits de décision et la répartition des responsabilités. Durant les trois périodes, le journal Libération a un profil proche du précédent, mais toujours avec une moindre intensité dans la mise en cause politique et morale. De leur côté, la presse économique (Les Échos, La Tribune) et Le Figaro privilégient l'approche économique tout au long du parcours. Leur question est de savoir dans quelle mesure cette opération financière et industrielle diffère ou non des négociations habituelles et des aléas boursiers qui les accompagnent. Enfin, le journal populaire Le Parisien utilise une approche toujours individualisante des situations. Les négociateurs, les responsables politiques et les traders sont toujours au premier plan. Si les arguments débutent dans le registre du "scandale », c'est l'angle de l'enquête policière et judiciaire qui est rapidement retenu et maintenu.

13 Cette analyse montre tout d'abord, données quantitatives à l'appui, que les différents répertoires disponibles sont utilisés dans les argumentations. Ceux en termes de "problème» (enjeux économique et industriel), d'«affaire " (une transgression à démontrer), et de «scandale " (une faute grave aux conséquences majeures) coexistent dans l'espace médiatique. Ensuite, pas plus que le dossier n'est par nature un « scandale », une "affaire » ou simple "problème ", chaque support médiatique modifie son mode d'argumentation dans la durée en fonction des informations qu'il collecte et des prises de position qu'il retient. Et comme cela s'observe souvent pour les dossiers complexes à caractère politico-financier, le registre du « scandale » qui prévaut en général au moment du lancement des mises en accusation publique, est progressivement réduit au profit des deux autres plus dédramatisants.

14 Ainsi, la scandalisation n'est au mieux qu'un moment du traitement social d'une situation problématique. Elle ne s'impose pas forcément d'entrée de jeu. L'évolution des discours montre surtout que les caractères transgressifs et les jugements normatifs et moraux qu'elle suppose perdurent rarement jusqu'au terme, même en cas de procès. Les déviances et délinquances des élites économiques et politiques bénéficient plus que d'autres de ces représentations euphémisées et réductrices des responsabilités. La même situation dans sa perception par un même acteur est fluctuante et les traits scandaleux ne sont qu'une qualification fragile et souvent temporaire, surtout s'il s'agit de délinquance économique ou politique. Louise Fines dans son travail sur Enron (2007) parvient à des 
résultats proches. Analysant la presse et les prises de position publique, elle montre comment, après la scandalisation initiale, le recours à la procédure de «négociation de plaidoyer $"{ }^{9}$ a provoqué une dilution des responsabilités et une minimisation des faits. Les interactions entre le service du procureur et les avocats de l'entreprise ont construit par étapes un dossier d'accusation basé sur des preuves judiciairement recevables avec, en contrepartie, le choix de charges allégées.

À terme, les effets sociaux de la mise en accusation publique sont souvent sans proportion avec la flambée émotionnelle et/ou idéologique initiale. Tout d'abord, la médiatisation produit rarement une bonne compréhension des mécanismes transgressifs à l'œuvre. Elle repose en général sur une schématisation et une individualisation des responsabilités. Pour Michael Levi (2006), la presse privilégie deux types d'interprétation des évènements : soit on a affaire à quelques "pommes pourries", soit "ils sont tous pourris ». Ensuite, les interprétations des faits et des décisions qu'ils supposent sont diverses. Ce qui fait «scandale » pour les uns ne le fait pas pour d'autres. Beaucoup de pratiques transgressives sont excusées, en tout ou partie. Les constructions discursives varient selon les valeurs et les stratégies des journalistes et de leur rédaction. On peut faire l'hypothèse que la même dynamique neutralisante s'observe chez beaucoup d'acteurs « experts » intervenant dans le débat public à de telles occasions.

\section{BIBLIOGRAPHIE}

ARMAND, Marie France, et Pierre LAScoumes (1977), « Malaise et occultation : perceptions et pratiques de contrôle social de la délinquance des affaires ", Déviance et Société, vol. $1, \mathrm{n}^{\circ} 2$, p. 135-169.

BLIC, Damiens DE (2003), « Le scandale à l'épreuve des sciences sociales », dans Le scandale financier, naissance et déclin d'une forme politique, de Panama au Crédit Lyonnais, thèse de sociologie, EHESS, chapitre 1, p. 22-62.

BLIC, Damiens DE (2005), « Moraliser l'argent. Ce que Panama a changé dans la société française (1889-1897) », Politix, 2005/3, nº 71, p. 61-82.

BLIC, Damiens DE, et Cyril LEMIEUX (2005), « Le scandale comme épreuve. Éléments de sociologie pragmatique ", Politix, 2005/3, nº 71, p. 9-38.

BOLTANSKI, Luc (1990) (dir.), «L'affaire comme forme sociale », dans L'amour et la justice comme compétence, Paris, Métailié, 1990, p. 255-271.

CLAVERIE, Élisabeth (1994), « Procès, affaire, cause. Voltaire et l'innovation politique », Politix, vol 7, $n^{\circ} 26$, p. 76-85.

DAMPIERRE, Éric DE (1954), « Thèmes pour l'étude du scandale », Annales. Économies, Sociétés Civilisations, vol. $9, \mathrm{n}^{\circ} 3, \mathrm{p} .328-336$.

FINES, Louise (2007), « Le jeu pénal insufflé par l'affaire Enron est-il en train de s'essouffler ? », Droit et société, $\mathrm{n}^{\circ} 2$, p. 451-462. 
GARRIGOU, Alain (1992), « Le boss, la machine et le scandale. La chute de la maison Médecin », Politix, vol. $5, \mathrm{n}^{\circ}$ 17, p. 7-35

HAMIDI, Camille (2009), « Le scandale n'aura pas lieu ou l'affaire Pechiney saisie par la presse », Revue française de sociologie, 2009/1, vol. 50, p. 91-121.

LASCOUMES, Pierre (1997) (dir.), « Scandale, affaire et problème », Élites irrégulières. Essai sur la délinquance d'affaires, Paris, Gallimard, p. 81-111.

LASCOUMES, Pierre (2013), « Élites délinquantes et résistance au stigmate. Jacques Chirac et le syndrome Téflon », Champ pénal, vol. X.

LEVI, Michael (2006), « The media construction of financial white-collar crime », British Journal of Criminology, vol. 46, $\mathrm{n}^{\circ}$ 6, p. 1037-1057.

OFFERLÉ, Michel (1998), «La scandalisation », Sociologie des groupes d'intérêt, Paris, Montchrestien, p. 122 et $\mathrm{s}$.

THOMSON, John B. (2000), Political Scandal, Cambridge, Polity.

TUMBER, Howard et Silvio R. WAISBord (2004), « Political Scandals and Media Across Democraties », Volume I, American Behavioral Scientist, vol. 47, $\mathrm{n}^{\circ}$ 8, p. 1031-1039.

TUMBER, Howard et Silvio R. WAISBORD (2004), « Political Scandals and Media Across Democraties », Volume II, American Behavioral Scientist, vol. 47, nº 9, p. 1143-1152.

\section{NOTES}

1. Une excellente revue de littérature a été réalisée sur ce sujet par Damien de Blic (2003 : 22-63).

2. Pour Thompson, cinq dimensions caractérisent le scandale : une transgression, le dévoilement d'une action dissimulée, une désapprobation collective, une publicisation, un effet de disqualification.

3. Voyez en ce sens par exemple Michel Offerlé (1998: 122 et s.) et Alain Garrigou (1992: 7 et s.).

4. Voyez par exemple Élisabeth Claverie (1994) et Damien de Blic (2003).

5. Ce dossier a mis en cause une entreprise d'État française spécialisée dans la production et le raffinage du pétrole. Il comporte de nombreux volets allant de l'enrichissement personnel des dirigeants, la tenue de caisses noires, et différentes formes d'abus des biens sociaux de l'entreprise tant pour des financements politiques que pour la rémunération de divers protégés.

6. Le Figaro, Libération, Le Monde, Le Parisien (journaux d'information générale), Les Échos et La Tribune (journaux d'information économique).

7. Logiciel conçu par Max Reinert. Il a l'intérêt de ne pas exiger de définition a priori des mots ou expressions importants. Il dégage des cooccurrences, des associations de termes qui définissent des champs sémantiques.

8. Titres de première page et en page intérieure.

9. Plea bargaining ou « plaider coupable ». 


\section{RÉSUMÉS}

Cet article démontre qu'aucune situation sociale de transgression n'a de propriété "naturelle», même si elle concerne les élites. Elle peut être perçue aussi bien comme un " problème ", comme une " affaire » ou comme un «scandale ». Chacune de ces qualifications sociales est le résultat de la combinaison de quatre variables : le degré de violation d'une norme; l'existence ou non de victimes et de dommages; le degré de responsabilité attribuée aux auteurs ; la réaction sociale attendue.

L'analyse empirique d'un dossier de presse concernant un cas de fraude boursière impliquant des acteurs politiques met en évidence trois phénomènes. Tout d'abord, dans l'espace médiatique il y a au même moment co-existence des différentes qualifications possibles du même évènement. Ensuite, l'évolution globale des discours sur une durée de six ans montre la diminution des arguments dénonçant un scandale politique au profit des arguments techniques et économiques. Enfin, l'évolution du contenu de chaque journal précise les transformations de leurs analyses en fonction de leur ligne éditoriale.

This article shows that no social situation of deviance has natural "properties", even when it concerns elites. It can be considered as a "problem", an "affair" or a "scandal". Each of those social qualifications is the result of four complementary variables: the degree of violation to the norm, the existence or not of victims and damage, the degree of attributed responsibility to the defendants, and the foreseen social reactions.

The empirical analysis of a press kit dealing with a case of insider trading fraud including political actors highlights three phenomena. First, different possible qualifications co-exist in the media on the same event. In addition, the global evolution of discourses on the course of six months illustrates the decrease in arguments denouncing a political scandal and the increase of economic and technical arguments. Finally, the evolution of the content of each newspaper clarifies their analytical changes according to their editorial lines.

\section{INDEX}

Mots-clés : médias, qualification sociale, dénonciation, dramatisation

Keywords : media, social qualification, denunciation, dramatization

\section{AUTEUR}

PIERRE LASCOUMES 